\title{
Effect of Structural Modification on Corrosion Behavior of Hypo Eutectic Al-Si Alloy
}

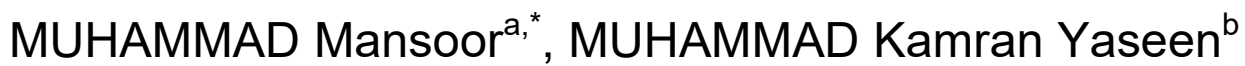 \\ and SHAHEED Khan ${ }^{\mathrm{C}}$ \\ Institute of Industrial Control Systems, Rawalpindi-PAKSITAN \\ amalik01677@yahoo.com, bkamran.met@gmail.com, 'shaheed_pe@hotmail.com
}

\section{Keywords: Al-Si Eutectic Alloy, Structural Modification, Pitting Corrosion Mechanism}

\begin{abstract}
Al-Si eutectic cast alloys are widely used in aeronautical and automobile industries where significantly high strength, toughness and wear resistance are required. This class of cast alloys exhibit relatively low corrosion resistance in brine environments. The mechanical properties of the alloy system mainly depend upon the shape of Si rich eutectic phase, which mainly has acicular geometry. In present research, the effect of modified microstructure of $12 \mathrm{wt}$ \% Si-Al alloy on corrosion behavior was studied. The needle like Si rich eutectic phase was modified to disperse spherical structure using rare earth metal halides. The corrosion rate and pitting behavior of modified and unmodified alloy were evaluated in $3.5 \% \mathrm{NaCl}$ solution by general corrosion for calculated time. It was observed that the corrosion rate and pitting tendency of modified alloy had been appreciably reduced as compare to unmodified alloy. The improvement of corrosion properties were the attributes of changed morphology and distribution of Si rich eutectic phase.
\end{abstract}

\section{Introduction}

The Al-Si alloy is a binary eutectic alloy having about $11-13 \%$ silicon. It is frequently used in aeronautical and automobile industries for cylinders, pistons, guiderails, etc. The composition of most $\mathrm{Al}-\mathrm{Si}$ foundry alloys is in the vicinity of the eutectic point $(12 \% \mathrm{Si})$ in order to take full advantage of its excellent castability. These alloys normally contain about 50 - 90 vol. \% (Al-Si) eutectic phase. The eutectic phase is important in determining the mechanical properties of these alloys [1].

In unmodified form, the eutectic silicon phase is brittle and has coarse and needle-like morphology. The sharp ends of this needle-like silicon phase serve as stress concentrators and initiate cracks and ultimately result in poor mechanical properties of the alloy. Through the process of modification, the brittle, coarse and needle-like eutectic silicon structure can be transformed to fine fibrous acicular eutectic silicon structure with much improved mechanical properties. Modification is the process of adding trace amounts of certain elements to an alloy to change the morphology of the phase(s) present in the alloy [2].

In present work, corrosion behaviors of the un-modified and modified alloys were investigated in $3.5 \% \mathrm{NaCl}$ aqueous solution. The corrosion test was carried out by submerging test specimens in the solution at ambient temperature for specified duration. Latter, the corrosion rates were calculated by weight loss method and corrosion behaviors were studies using microscopic examination. A surface energy investigations were also carried out to relate the variation of surface energies with the corrosion mechanisms of both the alloys.

\section{Experimental}

Alloy Making. An Al-Si hypo eutectic alloy was prepared using melt-cast route in an air melting furnace. The alloy contained 8-11 wt. \% of silicon as major alloying element besides the addition of 0.2-0.3 wt. \% magnesium, and 0.3-0.5 wt. \% of manganese. The impurities like iron, copper, and zinc were limited to less than $0.3 \mathrm{wt} \%$, individually. The detailed preparation of the alloy and subsequent modification are discussed elsewhere [3]. Briefly, the cleaned and preheated charge was 
melted in an electric melting furnace under protective flux. The melt temperature was kept at 735 to $745^{\circ} \mathrm{C}$, during which degassing was carried out using Degaser-190 tablets provided by FOSCECO, UK. After degassing, part of the melt was cast into $18 \mathrm{~mm}$ diameter rods, which represented unmodified alloy. While the remaining melt was treated with modifier salts to generate the modified alloy. Energy dispersed spectrometry (EDS) and optical spark emission (OSE) were used to analyze the composition of the alloys (Table 1). Further, microstructural features of the alloys were characterized using scanning electron microscope (SEM) in secondary electron mode, which confirmed the modification of the secondary silicon rich eutectic phase (Fig. 1).


Fig. 1 Morphology of the secondary silicon rich eutectic phase, a) unmodified needle like and

b) modified globular like phases. Insets are the respective micrographs at 1000X.

Table 1 Chemical composition of prepared alloy.

\begin{tabular}{l|l|l|l|l|l|l}
\hline Elements & Si & Mg & Mn & $\mathbf{C u}$ & Fe & Al \\
\hline \%age & $11.8 \pm 0.3$ & $0.44 \pm 0.21$ & $0.58 \pm 0.11$ & $0.07 \pm 0.02$ & $0.4 \pm 0.1$ & Balance \\
\hline
\end{tabular}

Surface Energy Measurements. The corrosion behavior of the specimens under investigations could be influenced by their surface energies. However, there is no direct method to determine surface energy of the solids. Therefore, sessile drop method was used to determine the contact angles using two different liquids i.e. water and ethylene glycol in accordance with ASTM 5946-04 standard. Latter, the investigations were extended to calculate surface energies of the specimens using Owens-Wendt method [4]. According to which:

$$
(1+\cos \theta) \gamma L=2[\sqrt{\gamma D L \times \gamma D S}+\sqrt{\gamma P L \times \gamma P S}]
$$

where; $\gamma_{\mathrm{PL}}$ and $\gamma_{\mathrm{DL}}$ are polar and dispersive component of the liquid, while $\gamma_{\mathrm{L}}$ is liquid surface energy. Similarly, $\gamma_{P S}$ and $\gamma_{D S}$ are polar and dispersive component of the solid surface. In present study, water and ethylene glycol were used to determine the contact angle, $\theta$. The respective surface energy, polar and dispersive components are 72.8, 51.0 and $72.8 \mathrm{~m} . \mathrm{Jm}^{-2}$ (for water) and 48.0, 19.0 and $29.0 \mathrm{~m} . \mathrm{Jm}^{-2}$ (for ethylene glycol).

Corrosion Testing. Effect of the modified silicon rich secondary phase on the corrosion characteristics of the alloy was studied using immersion corrosion test methods. The circular test specimens (20 mm diameter and $3 \mathrm{~mm}$ thickness) of each alloy were prepared and cleaned using 5\% nitric acid solution in accordance with ASTM G1-03 standard. The immersion corrosion tests were in brine solution at room temperature by following the specifications of ASTM-G31-72 testing standard, which were:

i) braine solution contianed 3.5 wt. \% of sodium choride,

ii) test temperature was $25^{\circ} \mathrm{C}$

iii)solution was un-aerated

iv)volume of the solution was $0.2 \mathrm{ml} / \mathrm{mm}^{2}$ of the specimens surface area

v) loss of solution due to evaporation was controlled by frequent addition of the brine solution

vi)time of corrosion test (hours) $=2000 /$ corrosion rate in mpy ( $>200$ hours) 
After the test duration, the specimens were washed using the mixture of $50 \mathrm{ml}$ ortho-phosphoric acid and $20 \mathrm{ml}$ chromic trioxide, which removed the loose corrosion products from the surface. A four decimal place digital weighing balance as used to determine weight loss of the test specimens after corrosion. Surface morphology of the tested specimens were studied using SEM in secondary electron imaging mode.

\section{Results \& Discussion}

Alloy Making and Modification. As mentioned earlier, specimens of the both unmodified and modified alloys were sectioned and prepared for microstructural study. A complete modification of silicon rich secondary phase was achieved (Fig. 1 a \& b). The modification process had not only transformed the silicon rich phase in to prolific dispersion of fine phase but also reduced the interdendritic spacing (Fig. 1b). For consideration, it should be noted that such type of refinement can also achievable through a rapid solidification rate; without using a modifier. However, the mechanisms behind the two processes are quite different with their effects on mechanical properties. During rapid solidification, aluminum solidifies rapidly than silicon due to its higher thermal conductivity and latent heat of fusion. At higher cooling rate the lead of aluminum becomes larger causing encasement of lagging silicon crystals, hence fine globular microstructure could be achieved.

Whereas, during chemical modification, the chemical modifiers act as poison to already growing atomic silicon layers by getting adsorbed onto their surface steps and kinks; preventing further growth of silicon, which not only limits the size of silicon rich phase but also limits the dendrite growth as well. Hence a finer structure is achievable with better mechanical properties [5].

Surface Energy Evaluation. As described earlier, the contact angle was determined by sessile drop method using two different liquids i.e. water and ethylene glycol (Fig. 2). Latter, the surface energies of the specimens were calculated using the Owens-Wendt method. The values of the surface energies were 18.92 and $27.91 \mathrm{~m} . \mathrm{Jm}^{-2}$ for unmodified alloy and the modified alloy, respectively. It could be seen that about $1 / 3^{\text {rd }}$ increase in surface energy was resulted due to the modification process, which in turn reflects that the surface is more reactive. It seems that the morphology and distribution of secondary silicon rich phase have influenced the surface energies.


Fig. 2 Formation of contact angles on unmodified and modified LM9 alloy using water and ethylene glycole.

Corrosion Testing. The general corrosion rates of the test specimens were calculated using weight loss method. The specimens were ultra-sonically treated for five minutes in ethanol and then washed in hot water to remove corrosion products. Latter, the specimens were dried in an oven $\left(50{ }^{\circ} \mathrm{C}\right)$ for two hours and weighed using a four decimal places digital weighing balance. The corrosion rates of un-modified and modified alloys were $155 \times 10^{-3}$ and $84 \times 10^{-3}$ mpy, respectively. The results imply that a reduction of more than $45 \%$ was instigated after modification of the secondary silicon phase. 
The surface and cross sections of the corrosion test coupons were studied using optical microscope and scanning electron microscope (SEM). In Fig. 3a, surfaces of the test coupons are shown after corrosion test at low magnification, where it is evident that the frequency and severity of the corrosion attack is much higher in unmodified alloy than in case of modified one. To reveal the features of the corrosion on the surface of the test coupons, SEM studies were carried out in secondary electron mode (Fig. 3 b \& c). It was observed that the corrosion pits were formed on the surface of both the alloys, however their nature/appearance was distinct. In un-modified alloy, multiple and narrow openings were formed (Fig. 3b), which were scattered throughout the surface. Contrarily the pits in modified alloy were quite a few and had wide openings (Fig. 3c). Moreover, in regions other than pitting, the surface oxide layer was absent from the un-modified alloy, while a thick and consistent oxide layer was present on the surface of modified alloy. This observation depicted the fact that the corrosion mechanism was distinct in either case, which will be discussed in proceeding section of "Corrosion Mechanism".

To find out the severity and penetration of corrosion pits, cross sectional samples were prepared and studied using optical microscope. In case of un-modified alloy, the type of pit openings was "narrow and deep", whereas the shape of the pit beneath the surface was "vertical microstructural orientation' type (Fig. 3d). In modified alloys, the type of pit openings was "wide elliptical", and the shape was "undercutting" (Fig. 3e).

Pit Evaluations. Pits formed after the corrosion test in both the alloys were evaluated for their distribution density, size and depth using ASTM G46 standard. Prior to the evaluations, the corroded specimens were cleaned and prepared for metallographic studies according to ASTM G1 and ASTM E3 standards, respectively. The results are given in Table 2, where it could be seen that the pitting features are ameliorated after the modification process i.e. less prone to pitting corrosion.

Table 2 Evaluation of the pits for un-modified and modified alloys.

\begin{tabular}{clccc}
\hline & \multicolumn{1}{c}{$\begin{array}{c}\text { Distribution } \\
\text { Density }\end{array}$} & Size & Depth \\
\hline & $\begin{array}{l}\text { ASTM } \\
\text { Rating }\end{array}$ & $\mathrm{A}-4$ & $\mathrm{~B}-2$ & $\mathrm{C}-3$ \\
& $\begin{array}{l}\text { Numerical } \\
\text { Values }\end{array}$ & $3.77 \times 10^{5} / \mathrm{m}^{2}$ & $0.75-1.85 \mathrm{~mm}^{2}$ & $1.2-1.8 \mathrm{~mm}$ \\
& $\begin{array}{l}\text { ASTM } \\
\text { Rating }\end{array}$ & $\mathrm{A}-2$ & $\mathrm{~B}-1$ & $\mathrm{C}-1$ \\
\hline
\end{tabular}

Corrosion Mechanism. The pitting evolution models proposed by E. McCafferty [6] were used to evaluate the mechanisms involved in the corrosion of un-modified and modified alloys. These models are developed on the bases of electrode kinetics, where surface energy play a major role. According to the models, the pitting corrosion in aluminum alloys can occur by dissolution of the surface oxide film or by penetration of chloride ions through the surface oxide film; depending upon the surface energy, whether low or high, respectively.

Through surface energy measurement, it was experimentally demonstrated that the un-modified alloy was lower in energy than the modified one, which defined the pitting corrosion mechanism during corrosion testing. It is known that the outermost surface oxide layer is covered with hydroxyl groups $(-\mathrm{OH})$ when immersed in aqueous solutions [7]. In case of un-modified alloy, where the isoelectric point was higher than the $\mathrm{pH}$ of the solution, the surface acquired a positive charge, according to Eq. 2:

$$
-\mathrm{MOH}+\mathrm{H}^{+}=-\mathrm{MOH}_{2}{ }^{+} .
$$


Consequently, the negatively charged chloride ions reacted with the surface oxide film and eventually dissolution of the film occurred, as observed during microscopic studies (Fig. 3b). The dissolution of surface oxide layer resulted in accelerated corrosion not only in the form of general corrosion but also pitting corrosion because more area was exposed to the chloride ions. Therefore, large number of pits with greater depth were formed in un-modified alloy.

Contrarily, in modified alloy the surface energy was higher, which lowered the surface isoelectric point than the $\mathrm{pH}$ of the solution resulting in a negative surface charge (Eq. 3):

$$
-\mathrm{MOH}+\mathrm{OH}^{-}=\mathrm{MH}^{-}+\mathrm{H}_{2} \mathrm{O} .
$$

In the presence of negative surface charge, the chloride ions did not react with the surface oxide but penetrated through it and reacted with the base metal to initiate the pitting (Fig. 3c). In this case, the surface oxide film acted as barrier to the pitting attack of the chloride ions as penetration is diffusion process, hence less number of pits with shallow depth were formed as compare to un-modified alloy.
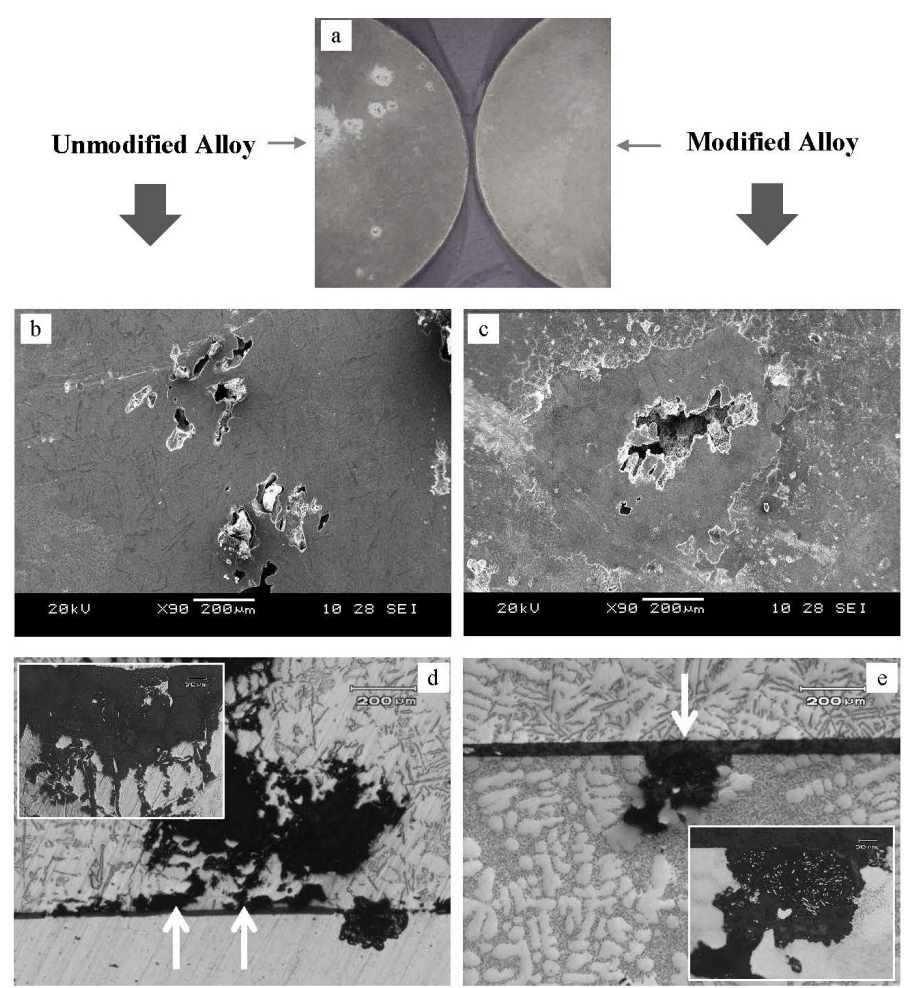

Fig. 3 (a) Stereo microscopic images of the test coupons after corrosion test, (b \& c) SEM micrographs showing pit opening morphologies at the surface and ( $\mathrm{d} \& \mathrm{e})$ optical micrographs showing type of pit and depth through the cross sections of un-modified and modified alloys.

\section{Conclusions}

Following conclusions were inferred:

- Modification process had increased the surface energy of the alloy from $18.92 \mathrm{~m} . \mathrm{Jm}^{-2}$ to 27.91 $\mathrm{m} . \mathrm{Jm}^{-2}$.

- Un-modified and modified alloys had corrosion rates of $155 \times 10^{-3}$ and $84 \times 10^{-3}$ mpy, respectively.

- Corrosion occured through the dissolution of the surface oxide film by chloride ions in unmodified alloy, while in modified alloy corrosion occured by the perentration of the chloride ions.

- Modified alloy had better corrosion chracteristic than un-modified alloy. 


\section{References}

[1] M.D. Hanna, S.Z. Lu, A. Hellawell, Metal. Mater. Trans. A, 15 (1984) 459-469.

[2] B.S. Pena, J.A. Lozano, Mat. Charact., 57 (2006) 218-226.

[3] M. Mansoor, I.u. Salam, A. Tauqir, Effect of microstructural modifier on the morphology of silicon rich secondary phase and strain hardening behavior of eutectic Al-Si alloy, Mat. Sci. Eng.: IOP conference Series, 146 (2016).

[4] D.K. Owens, R.C. Wendt, Estimation of the surface free energy of polymers, J. App. Polym. Sci., 13 (1969) 1741-1747.

[5] Vadims S. Zolotorevsky, Nikola A. Belov, Michael V. Glazoff, Casting Aluminum Alloys, Elsevier, Linacre House, Jordan Road, Oxford, UK, 2007.

[6] E. McCafferty, Sequence of steps in the pitting of aluminum by chloride ions, Corr. Sci. 45 (2003) 1421-1438.

[7] J.C. Bolge, Adhesion aspects of polymeric coatings, Plenum Press, New York,, 1983. 